Collection SFN 12 (2011) 263-283

(C) Owned by the authors, published by EDP Sciences, 2011

DOI: $10.1051 / \mathrm{sfn} / 201112013$

\title{
Experiments - Simulations - Theories: Multiscale approaches for solutions
}

\author{
J.-F. Dufrêche ${ }^{1,2}$, N. Malikova ${ }^{3}$, M. Jardat ${ }^{1}$, G. Mériguet ${ }^{1}$, E. Dubois ${ }^{1}$, \\ B. Rotenberg ${ }^{1}$, V. Marry ${ }^{1}$, J. Molina ${ }^{1}$ and P. Turq ${ }^{1,2}$ \\ 1 UMR PECSA, UPMC-Paris 6, CNRS, 4 place Jussieu, 75252 Paris Cedex 05, France \\ ${ }^{2}$ ICSM UMR 5257, CEA/CNRS/Université de Montpellier 2/ENSCM, Centre de Marcoule \\ Bât. 426, BP. 17171, 30207 Bagnols sur Cèze Cedex, France \\ ${ }^{3}$ Laboratoire Léon Brillouin, UMR CEA-CNRS 12, CEA Saclay, 91191 Gif-sur-Yvette, France
}

\begin{abstract}
The theory of complex media depends on various levels of description. At the macroscopic scale, hydrodynamic equations are used but they are not valid at small scales, the latter domain can be very important if slow processes occur in the system. At the microscopic scale, molecular dynamics describe the motion of the atoms individually. The two methods which correspond to different levels of description are complementary, but they are not the only ones. Intermediate methods based on continuous solvent model are able to take proper account of the solute dynamics and they can be applied at an intermediate time scale. We present such multi-scale descriptions in the case of solutions. Such an approach is all the more important in the context of of neutron science since neutron scattering experiments are now able to explore a large time domain: they are able to encompass the intermediate time domain for which intermediate methods are unavoidable.
\end{abstract}

\section{INTRODUCTION}

The description of the dynamics of complex systems, bulk or confined solutions, is to be made at different scales. At macroscopic scale, hydrodynamic equations are very convenient to model the convection motion and the diffusion of the various species present. Most of the time, the Navier-Stokes equation is coupled to the Fick's law in order to compute the macroscopic dynamic properties of the system as a function of the geometry. This final result can be compared to different experimental techniques. Among them, dynamic neutron scattering methods, such as neutron spin-echo or time of flight, very often play a central role because they are able to analyze the dynamics over a large time domain (from a few ps to $10 \mathrm{~s}$ of $\mathrm{ns}$ ), for which many transport processes can occur. For example the simple Fick's law can be analyzed from neutron scattering experiments, by determining the dynamic structure factor $S(q, t)$, which is, at the diffusion regime, equal to

$$
S(q, t)=\exp \left(-D q^{2} t\right)
$$

where $D$ is the diffusion coefficient. Yet the link among these macroscopic laws and the microscopic properties of the material is far from obvious. The hydrodynamic laws are valid only if the typical distances of the material are very large, but the exact condition has to be determined. In addition, macroscopic parameters (zeta potential, effective viscosity, diffusion coefficients, adsorption coefficients) are required in such macroscopic approaches.

A direct method to obtain this macroscopic quantities consists in deriving them from a molecular (atomistic) description. With the development of computer science, it is possible to perform molecular

This is an Open Access article distributed under the terms of the Creative Commons Attribution-Noncommercial License 3.0, which permits unrestricted use, distribution, and reproduction in any noncommercial medium, provided the original work is properly cited. 

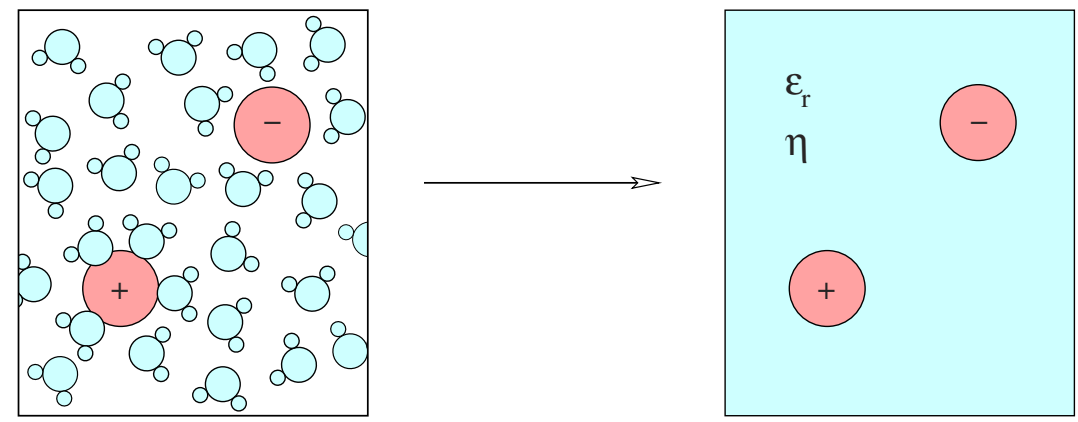

Figure 1. In discrete solvent models (left), the degrees of freedom of the solvent are treated explicitly. For continuous solvent models, the latter is considered as a continuum described by its dielectric constant $\epsilon_{r}$ and its viscosity $\eta$.

dynamics simulations in the case of complex systems. The precise motion of the particles is obtained at short time and short length scales. In that case the difficulty arises from the fact that the boundary conditions of the simulation do not correspond to the exact experimental system since the long-scale structure is ignored and the link with the experiments has to be clarified.

The two methods are complementary. They allow the description of the complex structure of the material. At short scales molecular dynamics can be used whereas macroscopic laws are fundamental at large scales. Yet, the link between the descriptions still requires further developments. Rigourous strategy can be obtained if one uses coarse-graining procedures where the microscopic laws are averaged over fast variables to get the long time macroscopic laws. We present such approaches here in the case of electrolyte solutions.

First, we will focus on continuous hydrodynamical models. We will briefly introduce the various levels of description that can be used in order to describe complex systems. Comparison between molecular dynamics and macroscopic laws allows the determination of the validity domain of commonly used theories, such as the Poisson-Boltzmann equation. In the case of hydrodynamics, the comparisons show that the macroscopic laws are valid if specific effects are taken into account.

Second, the case of ionic transport is analyzed. In that case, the classical atomistic descriptions (e.g. molecular dynamics) are not practically useful because of the long time scale of the ionic effects. Continuous solvent models, which can be rigourously derived from atomistic description are especially useful. In the case of simulations, Brownian dynamics can be performed to deal with ion dynamics at large scale. This continuous solvent scale is particularly interesting because the models are simpler so that explicit theories are possible in some cases. Such descriptions have been applied with success to bulk and confined solutions, the example of the latter being water and ions confined in clays.

\section{MULTI-SCALE DESCRIPTIONS}

\subsection{Discrete and continuous solvent models}

Most theories of electrolyte solutions are based on continuous solvent models. In that case (see Fig. 1), the complex nature of the solvent is simplified [1]. Instead of describing precisely the solvent molecules, the solvent is described as a continuum represented by global quantities, such as the dielectric constant $\epsilon_{r}$ or the viscosity $\eta$. In the same way, the ionic parameters depend on the solvent. The ion size is the hydrated size and for the dynamic properties the ionic mobility can be introduced [2, 3]. It represents the solvent friction around the ion.

Such continuous solvent models are very popular in the context of electrolyte solutions since the pioneering works by Debye, Hückel and Onsager $[4,5]$. The solvent properties are renormalized. The 
equilibrium or transport properties at infinite dilution, such as the solvation free energy, or the ion diffusion coefficient are difficulty to calculate from such approaches, because the molecular nature of the solvent drives an essential part of their value. Nevertheless, the concentration dependence of thermodynamic quantities (e.g. osmotic coefficient, ion activity) and of the ion transport coefficients (e.g. conductivity, mutual and self-diffusion coefficients) depends mainly on ion correlations [6], and these effects can be calculated satisfactorily by continuous solvent approaches.

\subsection{Coarse-graining procedure}

Continuous solvent models can be justified rigourously thanks to a coarse-graining procedure. Only the ions are explicitly taken into account: this class of models is valid if the solvent degrees of freedom can be treated independently of the solute motion. The situation is different for the equilibrium and the dynamical properties. In the case of equilibrium, the coarse-grained model is always justified. It is a direct consequence of statistical mechanics. The derivation has been performed by several authors, but probably the most relevant analysis is the one by McMillan and Mayer [7, 8].

The idea is to reduce the phase space from $\left\{\mathbf{r}_{\alpha}, \mathbf{p}_{\alpha}, \mathbf{r}_{i}, \mathbf{p}_{i}\right\}$ to $\left\{\mathbf{r}_{i}, \mathbf{p}_{i}\right\} . \mathbf{r}$ is the position and $\mathbf{p}$ is the momentum. $\alpha$ represents the solvent and $i$ stands for the solute particles. The relevant thermodynamical ensemble is the grand-canonical ensemble [9] for which the numbers of solvent and solute particles $N_{\alpha}$ and $N_{i}$ are not fixed but they are determined by the chemical potentials of the reservoir. The partition function reads [10]

$$
\begin{aligned}
\Xi\left(T, V, \mu_{\alpha}, \mu_{i}\right) & =\sum_{\text {états }} e^{\beta \mu_{\alpha} N_{\alpha}+\beta \mu_{i} N_{i}-\beta \mathcal{H}} \\
& =\sum_{N_{\alpha}=0}^{+\infty} \sum_{N_{i}=0}^{+\infty} \frac{e^{\beta \mu_{\alpha} N_{\alpha}+\beta \mu_{i} N_{i}}}{N_{\alpha} ! N_{i} ! h^{3\left(N_{\alpha}+N_{i}\right)}} \int \cdots \int e^{-\beta \mathcal{H}\left(\left\{\mathbf{r}_{\alpha}, \mathbf{p}_{\alpha}, \mathbf{r}_{i}, \mathbf{p}_{i}\right\}\right)} \mathrm{d} \mathbf{r}_{\alpha}^{N_{\alpha}} \mathrm{d} \mathbf{r}_{i}^{N_{i}} \mathrm{~d} \mathbf{p}_{\alpha}^{N_{\alpha}} \mathrm{d} \mathbf{p}_{i}^{N_{i}} \\
& =\sum_{N_{\alpha}, N_{i}} \frac{e^{\beta \mu_{\alpha} N_{\alpha}+\beta \mu_{i} N_{i}}}{N_{\alpha} ! N_{i} ! \lambda_{\alpha}^{3 N_{\alpha}} \lambda_{i}^{3 N_{i}}} \int \cdots \int e^{-\beta \mathcal{V}\left(\left\{\mathbf{r}_{\alpha}, \mathbf{r}_{i}\right\}\right)} \mathrm{dr}_{\alpha}^{N_{\alpha}} \mathrm{dr}_{i}^{N_{i}}
\end{aligned}
$$

where $\beta=1 /\left(k_{\mathrm{B}} T\right), T$ is the temperature, $V$ the volume, and $h$ is Planck's constant. $\mu_{\alpha}$ and $\mu_{i}$ are respectively the chemical potentials of the solvent and of the solute particles. $\mathcal{V}\left(\left\{\mathbf{r}_{\alpha}, \mathbf{r}_{i}\right\}\right)$ is the (molecular) interaction potential $V_{\text {ions }}\left(\left\{\mathbf{r}_{i}\right\}\right)+V_{\text {solvent }}\left(\left\{\mathbf{r}_{\alpha}\right\}\right)+V_{\text {solvent/ions }}\left(\left\{\mathbf{r}_{\alpha}, \mathbf{r}_{i}\right\}\right)$. $\lambda_{\alpha}$ et $\lambda_{i}$ are the solvent and solute de Broglie wave lengths ${ }^{1}$. They correspond to the position integration. The de Broglie length for a particle with mass $m$ reads $\frac{h}{\sqrt{2 \pi m k_{\mathrm{B}} T}}$. Here the calculation is given for a single solute, but the result is easily generalized if there are several different solutes. We finally get

$$
\Xi\left(T, V, \mu_{\alpha}, \mu_{i}\right)=\sum_{N_{\alpha}, N_{i}} \frac{z_{i}^{N_{i}}}{N_{i} !} \frac{z_{\alpha}^{N_{\alpha}}}{N_{\alpha} !} \cdots \int e^{-\beta \mathcal{V}\left(\left\{\mathbf{r}_{\alpha}, \mathbf{r}_{i}\right\}\right)} \mathrm{d} \mathbf{r}_{\alpha}^{N_{\alpha}} \mathrm{d} \mathbf{r}_{i}^{N_{i}}
$$

with $z_{i}$ and $z_{\alpha}$ the fugacities of the solute and of the solvent.

\footnotetext{
${ }^{1}$ For the phase space, we used the following discretization

$$
\sum e^{-\beta \mathcal{H}}=\cdots \int e^{-\beta \mathcal{H}} \frac{\mathrm{d} \mathbf{r}^{N} \mathrm{~d} \mathbf{p}^{N}}{N ! h^{3 N}} .
$$

It corresponds to the Heisenberg inequality $\Delta x \Delta p_{x} \sim h$. La correct relation would be more precisely $\Delta x \Delta p_{x} \sim h / 4 \pi$, but in the case of quantum perfect gas, the classical limit is actually recovered with $\Delta x \Delta p_{x} \sim h$. In any case, the difference between the two choices only modifies the definition of the de Broglie length. The resulting thermodynamical properties are the same, but a constant entropic term, which represents the reference for the standard part of the chemical potential. Thus the macroscopic thermodynamics is not modified.
} 
The Mc Millan and Mayer idea consists in factorizing the solute and the solvent parts:

$$
\Xi\left(T, V, \mu_{\alpha}, \mu_{i}\right)=\sum_{N_{i}} \frac{z_{i}^{N_{i}}}{N_{i} !} \cdots \int \mathrm{d} \mathbf{r}_{i}^{N_{i}} \sum_{N_{\alpha}} \frac{z_{\alpha}^{N_{\alpha}}}{N_{\alpha} !} \cdots \int e^{-\beta \mathcal{V}\left(\left\{\mathbf{r}_{\alpha}, \mathbf{r}_{i}\right\}\right)} \mathrm{d} \mathbf{r}_{\alpha}^{N_{\alpha}} .
$$

This expression is similar to the one of a single pure compound, if one considers that the solvent phase integration term is an effective potential, which acts between the solute particles. Practically we obtain

$$
\Xi\left(T, V, \mu_{\alpha}, \mu_{i}\right)=\Xi_{\mathrm{MM}}(T, V, a) \times \Xi_{\text {pure }}\left(T, V, \mu_{\alpha}\right)
$$

where $\Xi_{\text {pure }}\left(T, V, \mu_{\alpha}\right)$ is the partition function of the pure solvent when the solvent has the same chemical potential as in the solution. It corresponds to $\Xi\left(T, V, \mu_{\alpha}, \mu_{i}=-\infty\right)$. $\Xi_{\mathrm{MM}}(T, V, a)$ is the Mc MillanMayer partition function. Formally, it is the one of a pure simple fluid (without any solvent molecules):

$$
\Xi_{\mathrm{MM}}\left(T, V, a_{i}\right)=\sum_{N_{i}} \frac{a_{i}^{N_{i}}}{N_{i} !} \cdots \int e^{-\beta \mathcal{V}_{\text {eff }}\left(\left\{\mathbf{r}_{i}\right\}\right),} \mathrm{d} \mathbf{r}_{i}^{N_{\alpha}} .
$$

The effective potential between the solute particles is defined from

$$
\mathcal{V}_{\text {eff }}\left(\left\{\mathbf{r}_{i}\right\}\right)=-k_{\mathrm{B}} T \ln g_{N_{i}}\left(\left\{\mathbf{r}_{i}\right\}\right)
$$

where $g_{N_{i}}\left(\left\{\mathbf{r}_{i}\right\}\right)$ is the $N_{i}$-body correlation function of the solute in the limit $z \rightarrow 0$, i.e when the solute is infinitely dilute. The activity $a_{i}$ is an effective fugacity defined by

$$
a_{i}=z_{i} \lim _{z_{i} \rightarrow 0} \frac{C_{i}}{z_{i}}
$$

where $C_{i}$ is the equilibrium solute concentration. The chemical potential reads

$$
\begin{aligned}
\mu_{i} & =3 k_{\mathrm{B}} T \ln \lambda_{i}+k_{\mathrm{B}} T \ln z_{i} \\
& =3 k_{\mathrm{B}} T \ln \lambda_{i}+k_{\mathrm{B}} T \ln \gamma_{i}^{0}+k_{\mathrm{B}} T \ln a_{i}
\end{aligned}
$$

where $\gamma_{i}^{0}=\lim _{z_{i} \rightarrow 0} \frac{z_{i}}{C_{i}}$. Consequently, replacing $z_{i}$ by $a_{i}$ does not change the chemical potential, but one (constant) standard reference term (which corresponds to the Henry's law). This reference term corresponds to the conversion of the activity scale from the gas reference (solute in vacuum) to the solution reference (infinitely dilute solute).

This expression of the grand-canonical partition function allows the calculation of the various thermodynamical quantities. The grand potential $\Omega$ reads

$$
\begin{aligned}
\Omega & =-P V=-k_{\mathrm{B}} T \ln \Xi\left(T, V, \mu_{\alpha}, \mu_{i}\right) \\
& =-k_{\mathrm{B}} T \ln \Xi_{\text {pure }}\left(T, V, \mu_{\alpha}\right)-k_{\mathrm{B}} T \ln \Xi_{\mathrm{MM}}(T, V, a) \\
& =\Omega_{\text {pure }}+\Omega_{\mathrm{MM}} .
\end{aligned}
$$

$\Omega$ is the sum of the grand potential of the solvent and the grand potential of the McMillan-Mayer partition function. The McMillan-Mayer term is the one of a simple liquid (or gas) for which the phase space is only determined by the solute positions, with an effective potential given by eq. (2.7). It corresponds to a continuous solvent model for which the influence of the solvent is completely taken into account from the solvent-averaged potential between the solutes particles $\mathcal{V}_{\text {eff }}\left(\left\{\mathbf{r}_{i}\right\}\right)$.

The various equilibrium quantities are obtained thanks to the usual thermodynamical formulas. For example, if there are several solute species $i$ :

$$
\mathrm{d} \Omega=-P \mathrm{~d} V-S \mathrm{~d} T-N_{0} \mathrm{~d} \mu_{0}-\sum_{i} N_{i} \mathrm{~d} \mu_{i}
$$

by using index 0 for the solvent. For the pressure we obtain

$$
P=P_{\text {pure }}+P_{\text {osm }} \text {. }
$$


$P_{\text {pure }}$ is the pressure of the pure solvent in osmotic equilibrium with the solution, i.e. with the same chemical potential and temperature. $P_{\mathrm{osm}}$ is obtained from the McMillan-Mayer partition function. It is the osmotic pressure of the solution $[1,11]$ and it is equal to the difference between the pressure $P$ and $P_{\text {pure }}$.

Even if the McMillan-Mayer relations are exact, the quantities obtained from the partition function of the continuous solvent model are not directly the one measured from the experiments. A conversion between the McMillan-Mayer (MM) frame of reference to the experimental frame of reference ${ }^{2}$ is necessary. This conversion arises from the fact that in the McMillan-Mayer theory the derivations are performed at constant chemical potential of the solvent whereas in the experimental LR frame of reference, the thermodynamical quantities are defined at constant solvent concentration [12-16]. This conversion leads to a correction factor that is zero for a dilute solution. Globally, it is small for most of the electrolytes if they are relatively dilute (approximately $2 \%$ for most of the electrolytes at $1 \mathrm{~mol} \mathrm{~L}^{-1}$ ).

Thus, the McMillan-Mayer theory implies that it is always possible to theoretically model a solution with a continuous solvent model. In addition, the procedure is in principle exact, since it is a simple consequence of the fact that it is always possible to factorize a grand-canonical partition function. The role of the solvent is taken into account by the pure solvent term $\Omega_{\text {pure }}$, the solvation term $k_{\mathrm{B}} T \ln \gamma_{i}^{0}$ and the McMillan-Mayer (MM) effective potential $\mathcal{V}_{\text {eff }}\left(\left\{\mathbf{r}_{i}\right\}\right)$. However, practically, such an exact calculation is not possible. Indeed, the calculation of the effective potential is not easy. For example, it is not a pair-wise additive potential, even if the original microscopic potential is pairwise additive: the solvent-averaged force between two solute ions is perturbed by the presence of a third solute particle. The pairwise-additivity is recovered only in the case of dilute solution of which $N$-body effects are globally negligible. Thus, the continuous solvent models have been proved to be useful for dilute solutions. Within this limit, it is not too bad an approximation to account for the 2 particle terms. It is the low density limit. Most of the theories [1] do note calculate the MM potential but it is considered to be given by a model. The most popular one is the Primitive Model (PM) for which the ions are assumed to be charged hard spheres. The main advantage of such an approach is the fact that it leads to explicit expressions for the thermodynamical properties (free energy, structure factors, etc) if simple but accurate approximations are taken into account, such as the Mean Spherical Approximation (MSA) [17]. It is especially useful for chemical engineering, and for the interpretation of experiments.

The calculation of the effective potential between the ions from an atomistic description is actually possible. It can be carried out from Monte Carlo and molecular dynamics simulations [18-20]. The three-body effects are found to be negligible for simple electrolytes if the concentration is not too high (namely $c<2 \mathrm{~mol} \mathrm{~L}^{-1}$ roughly), the 2 particle effective potential reproducing well the basic features of the high-order structure. The ( 2 particles) effective potential between $i$ and $j$ reads

$$
V_{e f f}(r)=\frac{Z_{i} Z_{j} e^{2}}{4 \pi \epsilon_{0} \epsilon_{r} r}+V_{S R}(r)
$$

where the first term corresponds to the long range electrostatic interactions between two charges $Z_{i}$ and $Z_{j}, e$ is the elementary charge, $\epsilon_{0}$ is the permittivity of vacuum, $\epsilon_{r}$ is the dielectric constant of the solvent and $r$ is the distance between $i$ and $j$. The actual effective pair potential between two ions is therefore similar to the potential of the PM. Within the PM, the short range part $V_{S R}(r)$ is modelled by a hard sphere repulsion. It is worth noting that in fact [19] $V_{S R}(r)$ makes oscillations which reflect the molecular nature of the solvent, as it is represented in Fig. 2. Then the validity of the PM seems to be surprising. In fact, the situation is not that bad: at infinite dilution $V_{S R}(r)$ is negligible, the DebyeHückel theory being exact [21]. Thus the short range term is a kind of perturbation to the electrostatic term and it can be modelled by a simple hard sphere repulsion, as it can be shown from perturbation

\footnotetext{
${ }^{2}$ Which is commonly called the Lewis-Randall (LR) frame of reference.
} 


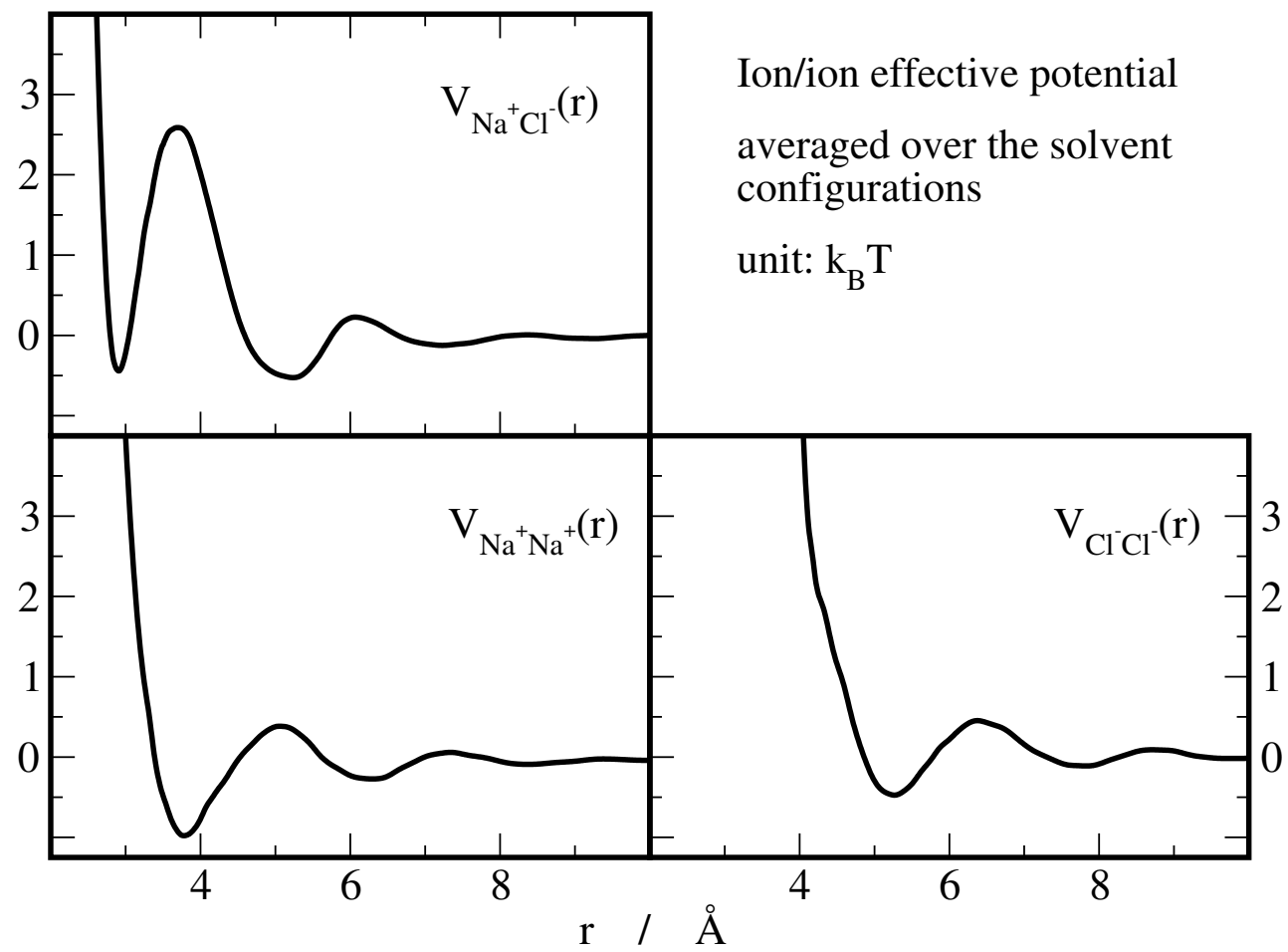

Figure 2. Interaction potentials between two ions in water obtained from molecular dynamics simulations at $25^{\circ} \mathrm{C}$ for a $\mathrm{NaCl}$ solution. Short range part $\left(V_{S R}(r)\right)$ only.

theory of fluids [22]. For very concentrated solutions this assumption does not hold, but anyway the effective potential between the ions is not pair additive anymore.

A rigourous procedure, similar to the McMillan-Mayer theory is not possible for the dynamical properties. Continuous solvent models are not exact for the dynamics. Yet, it is possible to justify such a coarse-grained approach for the dynamics using Brownian motion [23, 24]. If the solute particle are much heavier than the solvent molecules, one can consider that the dynamics of the solvent is much faster than the dynamics of the solutes. Then the solute particles move independently of the solvent molecules. They can be assimilated to Brownian particles. In that case, the dynamics of the solute is driven by equations of Brownian motion, which is a kind of continuous solvent model $[6,25,26]$.

The classical theories of electrolyte solutions are actually Brownian theories. The well known limiting laws by Debye, Hückel, and Onsager [5] for the transport properties of ions are Brownian motion theory results. For example, the Fuoss-Onsager equation, which has been originally derived by a phenomenological approach can be obtained from the Smoluchowski equation which is an equation for the Brownian motion [24, 26].

Strictly speaking, the Brownian motion approximation is valid only if the ions have a large mass relative to the mass of the solvent molecules [24]. This assumption is valid for heavy ions, such as $\mathrm{Cs}^{+}$, but it is a priori far from obvious for most of the simple ions. Yet, the Brownian approximation is practically valid for the dynamics of all ions - not only the heaviest. For example, Brownian theories successfully work for a very light ion such as $\mathrm{Li}^{+}$, even if the bare ion is actually much lighter than the solvent molecules [27].

It is difficult to show exactly why light ions are actually Brownian particles, but one can understand that it is related to hydration. The bare mass of an ion does not entirely drive the dynamics of the ion 


\begin{tabular}{|c|c|c|c|c}
$\begin{array}{c}\text { Ab Initio (Quantum) } \\
\text { Molecular Dynamics }\end{array}$ & $\begin{array}{c}\text { Classical } \\
\text { Molecular Dynamics }\end{array}$ & Brownian Dynamics & $\begin{array}{c}\text { Hydrodynamics } \\
\text { Non Eq. Thermodynamics }\end{array}$ & $\begin{array}{c}\text { Porous Media } \\
\text { Hydrodynamics }\end{array}$ \\
\hline $\begin{array}{c}\text { Nuclei } \\
\text { Electrons (density) }\end{array}$ & $\begin{array}{c}\text { Atoms Molecules } \\
\text { Ions }\end{array}$ & $\begin{array}{c}\text { Ions (Solute Particles) } \\
\text { Continuous Solvent }\end{array}$ & $\begin{array}{c}\text { Continuous Description } \\
\text { Navier-Stokes, Fick } \\
\text { Poisson-Bolzmann Eqs. }\end{array}$ & $\begin{array}{c}\text { Pore Averaged Laws } \\
\text { (e.g.: Darcy's Law) }\end{array}$
\end{tabular}

Figure 3. Various levels of description for the dynamics in bulk or confined solutions. Upper part : simulation and theoretical techniques. Lower part : ingredients of the models.

in a solution. Every ion is actually surrounded by a shell of solvent molecules. Thus it is probably not too bad an approximation to consider that the ion and the solvation molecules are a kind of effective particles which are much heavier than the solvent molecules so that it can be modelled by a Brownian approximation. It is not easy to demonstrate rigourously that idea, but the Brownian approximation is probably valid for electrolyte solutions because the ions are hydrated. In addition, the hydration is all the more important when the ion is small, because of the strong electric attraction, so that even small ions such as $\mathrm{Li}^{+}$can be modelled using a Brownian description.

\subsection{Scales of descriptions for solutions}

Multiscale descriptions of solutions can be performed. The various level of descriptions are summarized in Fig. 3.

Ab-initio molecular dynamics [28-30] provides information about the electronic degrees of freedom, but because of its computational cost it is restricted to the smallest time and length scales. Classical Monte-Carlo [31] or Molecular Dynamics simulations [32-34] are able to describe larger systems. Nevertheless, very large systems (e.g. for the calculation of the effect of the electric force, which is a long-ranged interaction) are hardly treated by this technique. The use of alternative methods such as Brownian dynamics simulations $[25,35,36]$ is inescapable. In that case, only a part of the degrees of freedom (e.g. the motion of the (free) ions) is considered. At large scales, continuous hydrodynamics methods (e.g. Poisson-Boltzmann [37] descriptions or Poisson-Nernst-Planck approaches [38-41]), which are based on non-equilibrium thermodynamics [42] can model the different dynamical effects. They are based on macroscopic hydrodynamic equations such as the Navier-Stokes equation or the Fick's law. They are especially relevant for the derivation of the global macroscopic law for the calculation of the various electrokinetic phenomena. For confined solutions, a more macroscopic method exists. Within that level of description, the solution has been averaged over the geometry of the confining porous systems, so that further laws (such as Darcy's law) have to be taken into account.

\subsection{Elimination of fast variables}

Unfortunately, the link among these methods is at first sight far from obvious. Yet this issue is very important since every method requires the knowledge of the parameters of the model it is based on. These parameters could be obtained - at least in principle - from the simulations at the lower level of description. This self-consistent approach is not commonly used, because of the time cost, though. Only a few attempts have been made in that direction (e.g. for clays [36, 43]). Most of the numerical studies use parameters obtained from experiments or from semi-phenomenological considerations.

The general method, which bridges the gap among the various models, is the elimination of fast variables [44]. Every level of description can be obtained from the preceding one by averaging over some degrees of freedom. Then it only describes the dynamics of a part of the degrees of freedom (relevant variables) and further degrees of freedom (irrelevant variables) are not described explicitly (on that level). The latter are only included via their average effect. For example, in the case of molecular 
dynamics, the only relevant variables are the positions of the nuclei. The details of the electronic degrees of freedom are ignored and only their average effects, i.e. the forces on the nuclei are taken into account. This separation among the variables makes sense for the calculation of the dynamical properties only if the irrelevant variables are much faster than the relevant ones. Thus, such a coarse-grained procedure is valid if slow variables coincide with relevant ones. In the case of molecular dynamics, it corresponds to the Born-Oppenheimer approximation that simplifies the calculation of the motion of the nuclei under the influence of the rapid motion of the electrons. Similarly, in the case of Brownian dynamics, the motion of the solute particles (i.e. ions) is obtained by considering that the only relevant variables are the degrees of freedom of the solute particles. This Brownian description which treats the solvent as a continuum is valid only if the solvent molecules are much faster than the solute particles.

The elimination of the fast variables is the key for the derivation of the various models of Fig. 3. The experimental macroscopic laws for confining porous media (such as Darcy's law), which are theoretically obtained differently (by averaging over the geometry of the material, for example thanks to the homogenization procedure of Moyne and Murad [40,41]) correspond to the research of slow variables.

\subsection{Hydrodynamic limit}

The hydrodynamic equations themselves correspond to the elimination of fast variables. Thus the laws of non-equilibrium thermodynamics [42] such as the Navier-Stokes equation for the convection and the Fick's law for the diffusive motion of the solute particles, are valid in the hydrodynamic limit [45, 46] where the hydrodynamic modes become slow. The latter corresponds to the limit of large distances $r$ or small wave-vectors $k$, as can be understood from the following argument.

Non-equilibrium thermodynamics predicts the evolution of particle and momentum distributions in space. For example, in the case of diffusion, it gives the evolution of the density of particles in space for the various components of the systems. The microscopic density of a species $\alpha$ reads microscopically:

$$
\rho_{\alpha}(\mathbf{r}, t)=\sum_{i} \delta\left(\mathbf{r}-\mathbf{r}_{i}(t)\right)
$$

where the $i$ th particle of type $\alpha$ has position $\mathbf{r}_{i}(t)$ at time $t$. The Fourier transform is :

$$
\rho_{\alpha}^{\star}(\mathbf{k}, t)=\int \rho_{\alpha}(t) e^{-i \mathbf{k} \cdot \mathbf{r}} \mathrm{d} \mathbf{r}=\sum_{i} e^{-i \mathbf{k} \cdot \mathbf{r}_{i}(t)}
$$

In the hydrodynamic limit $\mathbf{k} \rightarrow 0, \rho_{\alpha}^{\star}(\mathbf{k}, t)$ becomes time-independent because it tends towards $N_{\alpha}$, the total number of particles of type $\alpha$. For $\mathbf{k} \neq 0, \rho_{\alpha}^{\star}(\mathbf{k}, t) \neq N_{\alpha}$ depends on time. But its evolution becomes slower as $\mathbf{k} \rightarrow 0$. Thus for $\mathbf{k} \rightarrow 0$, the density of particles becomes a slow variable. A similar argument can be made for the average velocity (or momentum) of the particles:

$$
\mathbf{v}(\mathbf{r}, t)=\frac{1}{\sum_{i} m_{i}} \sum_{i} \delta\left(\mathbf{r}-\mathbf{r}_{i}(t)\right) m_{i} \mathbf{v}_{i}(t)
$$

because of the linear momentum conservation law. Thus in the hydrodynamic limit (small wave-vectors or large distances), hydrodynamic laws are valid because the hydrodynamic quantities become slow, on the grounds of the conservation laws.

The accuracy of hydrodynamic descriptions can be checked by comparing their predictions to the results obtained from molecular dynamics [47, 48]. Some of the results are given in Fig. 4 for $\mathrm{Na}$ montmorillonite clays with an interlayer spacing equal to $47.5 \AA$. In that case, macroscopic hydrodynamic models generally calculate counterion distributions with the help of the PoissonBoltzmann equation. Even for that relatively small interlayer spacing, this hydrodynamic model appears to be valid. The main differences are the oscillations given by the microscopic model, which are 


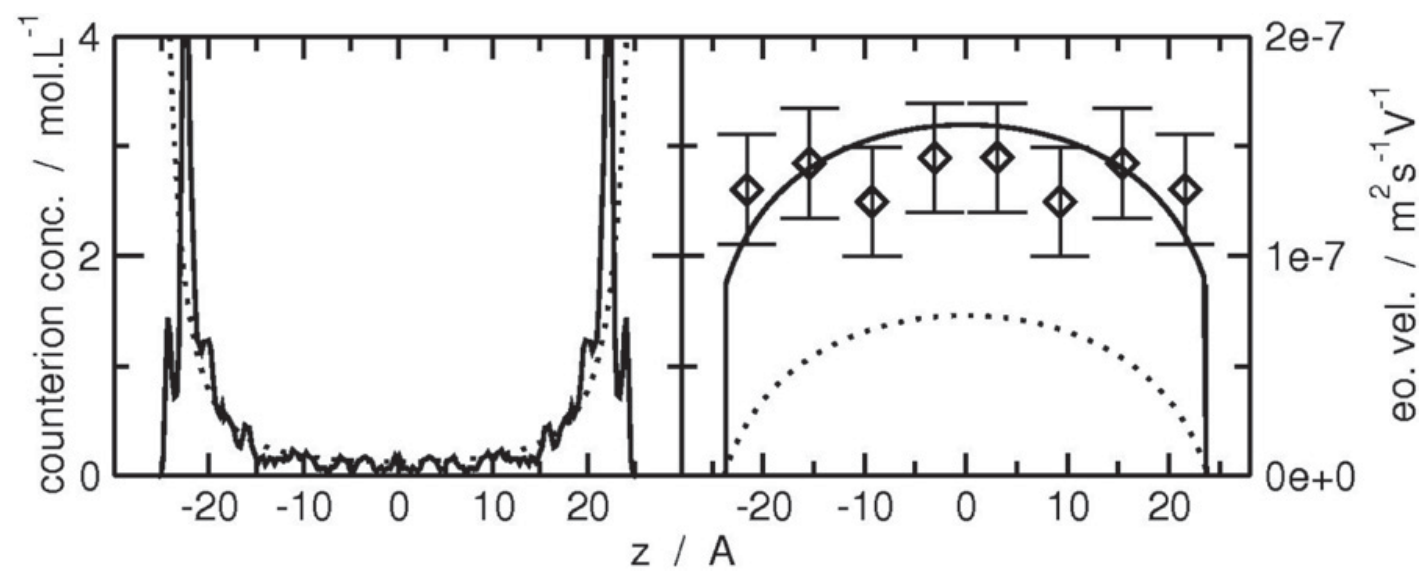

Figure 4. Left: Cation $\left(\mathrm{Na}^{+}\right)$distribution between two montmorillonite clay layers. Solid line: molecular dynamics. Dashed line : Poisson-Boltzmann equation. Right: Electro-osmotic flux. Diamonds: molecular dynamics. Dashed line: Navier-Stokes equation with stick boundary condition. Solid line: Navier-Stokes equation with slip boundary conditions $(\delta=6 \AA)$.

characteristic of the discrete nature of the solvent molecules. The electro-osmotic flow can be calculated from molecular dynamics as well. In that case simple hydrodynamic theory fails to reproduce the microscopic results. In fact, it seems that the problem comes from the stick boundary conditions. One can show that microscopically, slip boundary conditions have to be taken into account. More precisely, the parallel component of the hydrodynamic fluid velocity at the interface is not zero. It is actually given by:

$$
v_{p}=\delta \frac{\partial v_{p}}{\partial z}
$$

where $z$ is the direction orthogonal to the interface and $\delta$ is the Navier slip length that quantifies slipping. For montmorillonite, $\delta$ is equal to a few Ångströms, which is very small, but it is enough to modify the electro-osmotic flow. The reason comes from the fact that electro-osmosis is created by surface charges, so that it strongly depends on the boundary conditions. Conversely, hydrodynamic motions created by bulk forces (such as a pressure gradient in the case of a Poiseuille flow or of a streaming potential experiment) depend less on the slip length, as it has been recently confirmed by Joly et al. [49].

\section{DESCRIPTION AT THE INTERMEDIATE SCALE: BROWNIAN DYNAMICS AND CONTINUOUS SOLVENT MODELS}

\subsection{Brownian theories}

Most theories of transport of electrolyte solutions, which generalize the pioneering work of Onsager [5] are actually based on Brownian description. Different Brownian models can be implemented for the dynamics of ions in an aqueous solution. They are summarized in Fig. 5.

At the microscopic scale, discrete solvent descriptions consider all atoms, i.e. the ones of the solvent molecules and the ones of the solutes. The dynamics [10] is driven by Newton's law (which corresponds to the Liouville's equation in phase space).

$$
\frac{\mathrm{d} \mathbf{v}}{\mathrm{d} t}=\frac{\mathbf{F}}{m}
$$

where $\mathbf{F}$ is the force on the atom. Molecular dynamics solves this equation for all atoms of the solution. If solute particles can be considered to be much heavier than the solvent molecules, Brownian models 


\begin{tabular}{|c|c|c|c|c|}
\hline Model & $\begin{array}{c}\text { Microscopic } \\
\text { Variables }\end{array}$ & $\begin{array}{c}\text { Equation in the } \\
\text { Phase Space }\end{array}$ & $\begin{array}{c}\text { Equation of the } \\
\text { Trajectories }\end{array}$ & $\begin{array}{c}\text { Simulation } \\
\text { Method }\end{array}$ \\
\hline \hline $\begin{array}{c}\text { Discrete } \\
\text { Solvent }\end{array}$ & $\begin{array}{c}\left\{\mathbf{r}_{\text {solvent }}, \mathbf{p}_{\text {solvent }}\right. \\
\left.\mathbf{r}_{\text {solute }}, \mathbf{p}_{\text {solute }}\right\}\end{array}$ & Liouville & Newton & $\begin{array}{c}\text { Molecular } \\
\text { Dynamics }\end{array}$ \\
\hline $\begin{array}{c}\text { Continuous } \\
\text { Solvent } \\
m_{\text {solvent }}<<m_{\text {solute }}\end{array}$ & \begin{tabular}{c}
$\left\{\mathbf{r}_{\text {solute }}, \mathbf{p}_{\text {solute }}\right\}$ \\
\cline { 2 - 5 }
\end{tabular} & Fokker-Planck & Langevin & $\begin{array}{c}\text { Langevin } \\
\text { Brownian Dynamics }\end{array}$ \\
\hline
\end{tabular}

Figure 5. Various levels of description for the description of the dynamics of ions in solution.

(based on continuum solvent descriptions) are valid. The separation of timescales between the slow solute (ion) and fast solvent (water) dynamics is such that one can average over the solvent degrees of freedom. There are mainly two classes of Brownian description [24]. The first one corresponds to the Langevin approach. The relevant observables are the velocity (or the momentum) and the position of the ions. The evolution in phase space is given by a Fokker-Planck equation, as it can be shown by a Kramers Moyal expansion [50, 51]. The second one is the Smoluchowski approach. It is valid for time scales longer than the relaxation time $\tau_{\mathrm{v}}=1 / \gamma$ of the velocities of the ions. Indeed in that case, the ionic velocities have the time to relax and it can be shown [44] that they are actually fast variables. The dynamics of the ions is then given by the Smoluchowski equation, which only depends on the position of the particles.

Within the Langevin approach, the solute/solvent interaction is modelled by two parameters. First, the dynamical properties depend on a single friction coefficient $\gamma$ (in $\mathrm{s}^{-1}$ ): The friction force on an ion is proportional to its velocity $\left(\mathbf{F}_{\text {solv }}=-m \gamma \mathbf{v}\right)$. The important point is that $\gamma$ represents the dissipative force of the solvent, which depends on several dissipation processes. One of them is the hydrodynamical friction, which can be estimated from the Stokes relation $\mathbf{F}_{\text {solv }}^{\text {hyd }}=-6 \pi \eta R \mathbf{v}$ with $\eta$ the viscosity of the solvent and $R$ the hydrodynamic radius of the solute particles. Another process is the so-called dielectric friction $[3,52,53]$ which represents the electrostatic friction for the solvent. $\mathbf{F}_{\text {solv }}^{\mathrm{DF}} \approx-Z^{2} e^{2}\left(\epsilon_{r}-1\right) \tau_{D} / R^{3} \epsilon_{r}\left(1+2 \epsilon_{r}\right) \mathbf{v}$ with $\tau_{D}$ de Debye relaxation time of the solvent molecules. The dielectric friction is significant for small ions. The second parameter is the external (free energy) potential $V_{\text {eff }}$ which models the force on the ions averaged over the solvent configurations, and it is assimilated to the MM effective force.

The Langevin equation reads

$$
\frac{\mathrm{d} \mathbf{v}}{\mathrm{d} t}=-\gamma \mathbf{v}+\frac{\mathbf{F}}{m}+\Gamma(t)
$$

where $\mathbf{F}$ is the external force, i.e. the opposite of the gradient of $V_{\text {eff }} . \boldsymbol{\Gamma}(t)$ is a random force. It can be modelled by a white noise the correlation function of which is related to $\gamma$ [50]. More precisely, the noise is supposed to be isotropic $\langle\boldsymbol{\Gamma}(t)\rangle=\mathbf{0}$ and rapidly uncorrelated $\left\langle\Gamma^{\alpha}(t) \Gamma^{\beta}(t+\tau)\right\rangle=2 k_{\mathrm{B}} T m \gamma \delta(\tau) \delta_{\alpha \beta}$. This equality is the so-called fluctuation-dissipation theorem, which gives a link between the fluctuating force $\boldsymbol{\Gamma}(t)$ and the dissipation force $-\gamma \mathbf{v}$. It guarantees that the velocity fluctuations satisfy the Boltzmann law $\frac{1}{2} m\langle\mathbf{v}(t)\rangle=\frac{3}{2} k_{\mathrm{B}} T$.

The Langevin equation is equivalent to the Fokker-Planck (FP) equation

$$
\partial_{t} f+\mathbf{v} \cdot \nabla f=\nabla_{\mathbf{v}} \cdot\left(\gamma \mathbf{v}+\frac{\nabla V}{m}+\frac{\gamma k_{B} T}{m} \nabla_{\mathbf{v}}\right) f
$$

which gives the evolution of the probability density function $f(\mathbf{x}, \mathbf{v}, t)$ of finding an ion at a given position with a given velocity. The direct numerical solution of the FP equation can be obtained by a Lattice Fokker-Planck method $[54,55]$. It is especially adapted to the case of one solute particle 
in an external field. If there are several solute particles, Brownian dynamics at the Langevin scale is unavoidable.

A few studies have been made at the Langevin level of description [35]. In fact almost all theories and simulations correspond to the Smoluchowski level of approximation $[6,56,57]$. The reason is that the characteristic time of ions in bulk solution is the Debye time of the electrolyte $[58] \tau_{D}$.

$$
\tau_{\mathrm{D}}^{-1}=4 \pi L_{\mathrm{B}} \sum_{i} Z_{i}^{2} C_{i} D_{i}
$$

where $L_{\mathrm{B}}=e^{2} / 4 \pi \epsilon_{0} \epsilon_{r} k_{\mathrm{B}} T$ is the Bjerrum's length, and $Z_{i}, C_{i}, D_{i}$ are respectively the charge, concentration and diffusion coefficient at infinite dilution of the particles $i . D_{i}=k_{\mathrm{B}} T / m_{i} \gamma_{i}$. This time is typically of a few nanoseconds, it is much longer than the relaxation time of the velocities (closer to the picosecond) so that the Smoluchowski approach is valid. In that case, only the positions $\mathbf{r}$ of the particles are known. The equation of motion [59] reads

$$
\frac{\mathrm{d} \mathbf{r}}{\mathrm{d} t}=\frac{\overline{\mathbf{D}} \mathbf{F}}{k_{\mathrm{B}} T}+\mathbf{R}(t)
$$

The first term is the drift term. It depends on the diffusion matrix $\overline{\mathbf{D}}=\left(\begin{array}{ccc}D & 0 & 0 \\ 0 & D & 0 \\ 0 & 0 & D\end{array}\right)$ with $D=k_{\mathrm{B}} T / m \gamma$. The second term $\mathbf{R}(t)$ represents a random displacement. It is related to $\overline{\mathbf{D}}$ by a fluctuation-dissipation theorem. The relation (3.5) can be obtained from the Langevin equation in the limit $\gamma \rightarrow+\infty$. If there are several particles, hydrodynamic interactions appear. They represent the fact that the friction depend on the surrounding solute particles, because their motion change the motion of the solvent and consequently the friction on the solute particles. They are taken into account at the Smoluchowski level of approximation via the diffusion matrix $\overline{\mathbf{D}}$, which is not diagonal anymore. Several expressions of $\overline{\mathbf{D}}$ have been introduced. For a relatively dilute solution, the most commonly used is the Rotne-Prager tensor [60-62].

Brownian dynamics simulations [25] solve numerically this stochastic equation (3.5). The simplest algorithm is the Ermak's algorithm

$$
\mathbf{r}(t+\Delta t)=\mathbf{r}(t)+\left(\frac{\overline{\mathbf{D}} \mathbf{F}}{k_{\mathrm{B}} T}+\frac{\partial}{\partial \mathbf{r}} \cdot \overline{\mathbf{D}}\right) \Delta t+\mathbf{R}(t) .
$$

The second part $\frac{\partial}{\partial \mathbf{r}} \cdot \overline{\mathbf{D}}$ of the drift term comes from the hydrodynamic interactions and it is globally zero in most of the cases, because of the incompressibility of the solvent. The random displacement $\mathbf{R}$ is calculated at any time step by a random number generator which satisfies the following fluctuationdissipation conditions $\langle\mathbf{R}(t)\rangle=\mathbf{0}$ and $\left\langle\mathbf{R} \mathbf{R}^{T}\right\rangle=2 \overline{\mathbf{D}} \Delta t$.

\subsection{Equilibrium properties: application to ferro-fluids}

The Ermak algorithm (3.5) is equivalent to the Smoluchowski equation, which yields the evolution of $\mathcal{P}\left(\left\{\mathbf{r}_{i}\right\}, t\right)$, the probability density of the configuration $\left\{\mathbf{r}_{i}\right\}$ of the solute positions at time $t$. It is a continuity equation

$$
\frac{\partial \mathcal{P}}{\partial t}+\operatorname{div} \mathcal{P} \mathbf{U}=0
$$

with

$$
\mathbf{U}=\left(\begin{array}{l}
\mathbf{U}_{1} \\
\vdots \\
\mathbf{U}_{N}
\end{array}\right)
$$


$\mathbf{U}$ is a $3 N$ components vector ( $N$ is the number of solute particles). It represents the hydrodynamic velocity of the particles. It is related to the force $\mathbf{F}$ on the particles through $[63,64]$

$$
\mathbf{U}=\beta \overline{\mathbf{D}} \mathbf{F} \text {. }
$$

where $\overline{\mathbf{D}}=\overline{\mathbf{D}}\left(\left\{\mathbf{r}_{i}\right\}, t\right)$ is the diffusion tensor of the Ermak algorithm, $\beta=1 / k_{\mathrm{B}} T$. F corresponds to the force on the particles averaged over the solvent configurations

$$
\mathbf{F}=\mathbf{F}_{\mathrm{ext}}+\mathbf{F}_{\mathrm{int}}-\frac{1}{\beta} \operatorname{grad} \ln \mathcal{P}
$$

where $\mathbf{F}_{\text {ext }}$ is the external force applied on the solute particles (e.g. gravity or an external electric force). The internal force $\mathbf{F}_{\text {int }}$ can be identified with the McMillan-Mayer force. The third term $-\frac{1}{\beta} \operatorname{grad} \ln \mathcal{P}$ represents the diffusion force and it corresponds to the random displacement $R$ of the Ermak algorithm.

Equations (3.7), (3.9) and (3.10) are the Smoluchowski equations for a set of solute particles. If the solutes have internal degrees of freedom, internal variables have to be added, but the general form of the Smoluchowski equation is the same.

If internal and external forces are conservative forces, $\mathbf{F}=-\operatorname{grad} \phi-\frac{1}{\beta} \operatorname{grad} \ln \mathcal{P}$, as it is the case for the internal McMillan-Mayer force. Then it can be shown that the canonical distribution

$$
\mathcal{P}=A e^{-\beta \phi}
$$

is a stationary solution of the Smoluchowski equation. Thus the equilibrium of the system is simply given by the canonical distribution. It does not depend on the choice of the hydrodynamic tensor $\overline{\mathbf{D}}$.

The fact that the canonical law is recovered means that Brownian dynamics can be performed in order to obtain the equilibrium properties of the solution. In that case, Brownian dynamics [65] is a kind of "smart" Monte-Carlo simulation for which the equilibrium state is quickly obtained because the random fluctuation force is calibrated to go preferentially to the energy minima. This method has been used for example in the case of ferrofluids [66]. A suspension of spherical magnetic particles can be described by Brownian dynamics. In the modelled system, the nanoparticles are monodisperse with a diameter of $13 \mathrm{~nm}$ in order to recover the same dipolar interaction as in the real system. The magnetization of the material is the experimental value $3.1 \times 10^{5} \mathrm{~A} \cdot \mathrm{m}^{-1}$. The dipole moment is linked to the axis of the nanocrystal, which corresponds to a rigid dipole. The interparticle interaction has a dipolar anisotropic part and an isotropic part [67], the latter being modelled by a Yukawa potential which represents a DLVO force [68]. Parameters are estimated from a numerical adjustment of the structure factor of a concentrated dispersion (packing fraction $=10 \%$ ) measured using small angle neutron scattering (SANS) at $T=298 \mathrm{~K}$. The cubic simulation box with periodic boundary conditions contains 500 or 1000 particles, in a medium reproducing water at $298 \mathrm{~K}$. An Ewald summation allows the evaluation of the long ranged dipolar interactions. Simulations are performed with and without an external magnetic field. The resulting $2 \mathrm{D}$ structure factor, compared to the one measured from neutron scattering, is represented in Fig. 6.

The peak is sharper in the direction perpendicular to the field and smoother in the direction parallel to the field. This means that the solution is better structured perpendicular to the field than parallel to it. This is observed both for the calculated and the measured $S(q)$, as can be seen directly in Figure 6 . However, the amplitude of the variation is larger in the experiment than in the calculation, which may result from the polydispersity of the nanoparticles in the real system.

\subsection{Transport properties}

Brownian dynamics can be used for the calculation of the ion transport properties too. However, an alternative method, based on explicit (analytical) calculation is possible for simple electrolytes, by using the Primitive Model (PM). The calculation of transport coefficients is based on an accurate description of equilibrium. For electrolyte solutions, the PM can be solved by different techniques based for example 


\section{Small angle neutron scattering}

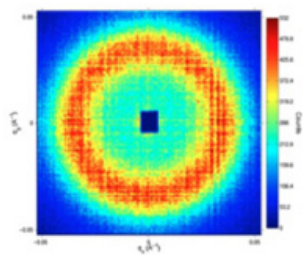

$H=0$

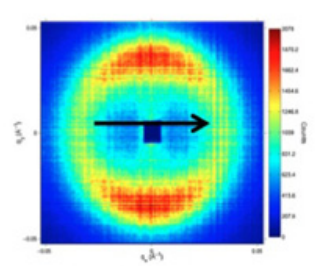

$H=8 \mathrm{kG}$

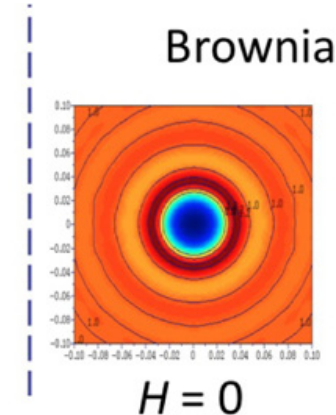

\section{Brownian Dynamics}

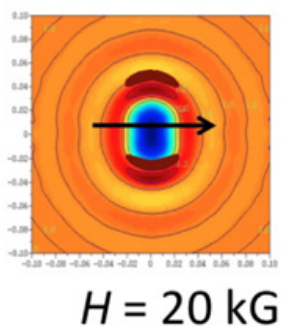

Figure 6. Structure factor of a suspension of ferrofluids. The (average) size of the particles is $13 \mathrm{~nm}$. The volume fraction is close to $10 \%$. The structure factor is obtained from SANS experiments (left) and Brownian dynamics simulations (right) for different values of the external magnetic field.

on integral equations [9, 10]. Among these methods, the Mean Spherical Approximation (MSA) is especially interesting since it yields to analytical formula [17, 69-72]. MSA generalizes the DebyeHückel theory with a screening parameter which is not anymore the inverse Debye length $\kappa_{\mathrm{D}}$ but $\Gamma$. $\Gamma$ is defined implicitly by

$$
\Gamma=\left[\pi L_{\mathrm{B}} \sum_{i} C_{i}\left(\frac{Z_{i}}{1+\Gamma \sigma_{i}}\right)^{2}\right]^{\frac{1}{2}}
$$

where $C_{i}$ (SI unit: $\mathrm{m}^{-3}$ ) and $\sigma_{i}$ are respectively the concentration and the hydrated diameters of ion $i$. $L_{\mathrm{B}}=e^{2} / 4 \pi \epsilon_{0} \epsilon_{r} k_{\mathrm{B}} T$ is Bjerrum's length. Thus, $2 \Gamma$ generalizes $\kappa_{\mathrm{D}}=\left(4 \pi L_{\mathrm{B}} \sum Z_{i} C_{i}^{2}\right)^{1 / 2}$. The inverse Debye screening length is replaced by $2 \Gamma$ because we account for the size of the ions in the ionic atmosphere. It is possible to get a simple but satisfactory theory of electrolyte solutions up to molar concentrations with the help of the MSA. The only parameters are the ion diameters. They can be obtained from equilibrium measurements (e.g. osmotic coefficients) [73, 74].

Then it is possible to calculate the various transport coefficients from the Smoluchowski equation $[26,27,75,76]$ in agreement with Brownian dynamics simulations. The method is based on a hierarchy of equations for the Smoluchowski equation, similar to the BBGKY hierarchy for the Liouville equation [10]. The final result is fully analytical. There are no fitted parameters since the sizes are known from equilibrium measurements. The ions are dynamically characterized by their friction (or their diffusion coefficient) at infinite dilution. The result for the conductivity and the mutual diffusion coefficients of several electrolytes is represented in Fig. 7. The predictions are in good agreement with the experimental values for simple electrolytes. The two important effects are the relaxation forces, which slow down the ions, and the hydrodynamic interactions.

\subsection{Time-dependent properties: Ion diffusion and mode-coupling theory}

Brownian approaches are also interesting for the description of time dependent transport, a phenomenon which can be measured thanks to neutron scattering experiments.

The ion self-diffusion coefficients (i.e. the diffusion coefficient of a tracer ion in the electrolyte solution) can be obtained similarly from the MSA [77]. For dilute solutions, the hydrodynamic interactions (HIs) are negligible. They decrease the relaxation force, and this second order effect can be neglected up to $1 \mathrm{~mol} \mathrm{~L}^{-1}$ for simple electrolytes, as it can be shown from Brownian dynamics simulations [25]. But the calculation of the relaxation force based on the Fuoss-Onsager equation is more complicated because the tracer is actually a further species. An alternative approach is modecoupling theory (MCT) $[78,79]$ which can be related to the Smoluchowski equation formalism. It yields the time-dependence of the diffusion process. 

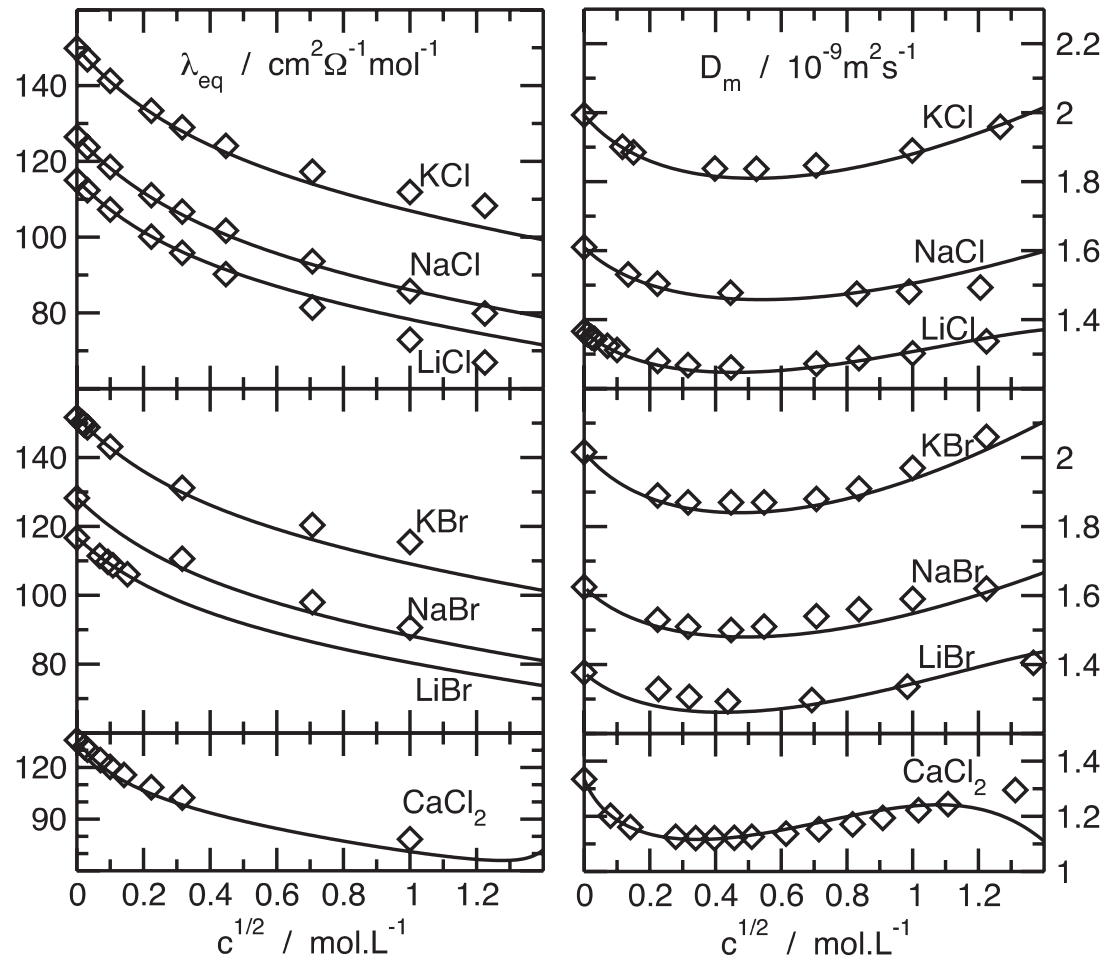

Figure 7. Equivalent conductivity and mutual diffusion coefficients of simple aqueous electrolyte solutions at $25^{\circ} \mathrm{C}$ from continuous solvent theory as functions of the square root of the concentration.

The Smoluchowski equation describes the system in the ion configuration space. The velocities of the particles are considered as fast variables and they have been eliminated. Thus, the classical Kubo formula $[10,80]$ for the self-diffusion coefficient

$$
D_{i}^{s}=\int_{0}^{+\infty} \frac{1}{3}\left\langle\mathbf{v}_{i}(t) \cdot \mathbf{v}_{i}(0)\right\rangle \mathrm{d} t=\int_{0}^{+\infty} Z(t) \mathrm{d} t
$$

has to be generalized to the Smoluchowski equation formalism [25, 63] because the velocity of the particles is not a parameter at the Smoluchowski level of description.

If the hydrodynamic interactions are neglected, the corresponding linear-response theory formula for the self-diffusion coefficient of species $i$ reads:

$$
D_{i}^{s}=D_{i}^{0}-\frac{1}{3} \int_{0}^{\infty} \mathrm{d} t\left(\frac{D_{i}^{0}}{k_{\mathrm{B}} T}\right)^{2}\left\langle\mathbf{F}_{i}(0) \cdot \mathbf{F}_{i}(t)\right\rangle,
$$

where $\mathbf{F}_{i}(t)$ is the force on particle $i$ at time $t$. The calculation of $D_{i}^{s}$ requires the calculation of the time dependent friction

$$
\delta \zeta(t)=\frac{1}{3 k_{\mathrm{B}} T}\left\langle\mathbf{F}_{i}(0) \cdot \mathbf{F}_{i}(t)\right\rangle .
$$

which can be calculated from a MCT approach [78, 79].

There is an alternative method which can be used to demonstrate (3.14). Instead of using the Smoluchowski equation, it is possible to start from the Langevin equation, which describes the motion 


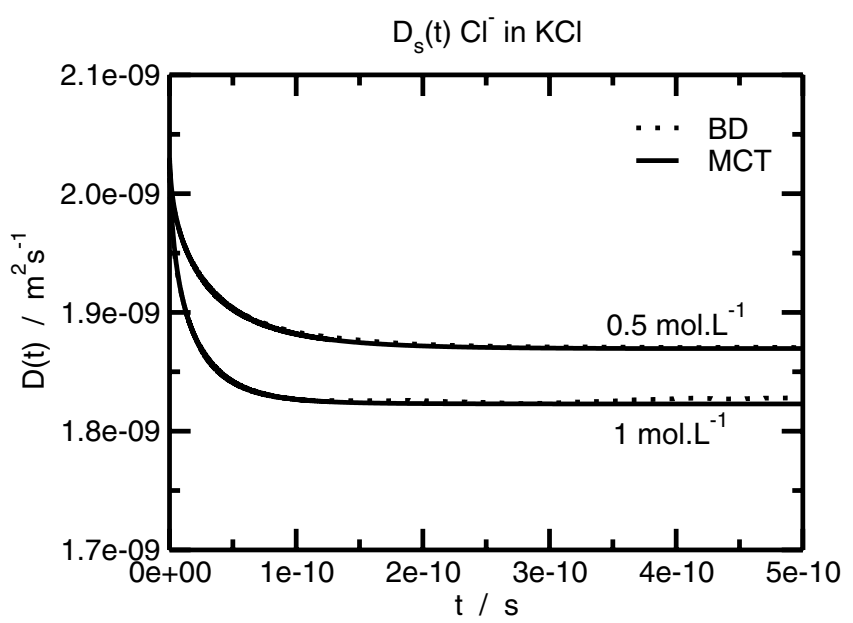

Figure 8. Time-dependent diffusion coefficient of chloride ion in aqueous $\mathrm{KCl}$ solutions at $25^{\circ} \mathrm{C}$ calculated by Brownian dynamics (BD) and Mode-Coupling Theory (MCT).

of the solute particles. When no HIs are considered, we have

$$
m \frac{\mathrm{d} \mathbf{v}_{s}}{\mathrm{~d} t}=-\zeta^{0} \mathbf{v}_{s}+\mathbf{F}_{i}^{M M}(t)+\mathbf{f}_{s}(t)
$$

where $\mathbf{v}_{s}$ is the velocity of the tracer particle and $\mathbf{f}_{s}(t)$ is the random force which represents the collision with solvent molecules. It evolves much faster than the other forces, so it can be modelled by a Gaussian white noise which satisfies the fluctuation-dissipation theorem $\left\langle\mathbf{f}_{s}(t) \cdot \mathbf{f}_{s}(0)\right\rangle=6 k_{\mathrm{B}} T \zeta^{0} \delta(t)$. The Langevin equation (3.16) being linear, it is possible to calculate $\mathbf{v}_{s}(t)$ as a function of $\mathbf{F}_{s}^{M M}(t)$ and $\mathbf{f}_{s}(t)$. Then, by averaging over the initial position of the tracer particle we get the velocity correlation function. More precisely, a straightforward calculation shows that

$$
Z(t)=\frac{k_{\mathrm{B}} T}{m} e^{\zeta^{0} t / m}-\frac{1}{3 \zeta^{2}}\left\langle\mathbf{F}_{s}^{M M}(t) \cdot \mathbf{F}_{s}^{M M}(0)\right\rangle .
$$

Since $1 / \zeta^{0}=D_{s}^{0} / k_{\mathrm{B}} T$ and $D_{s}=\int_{0}^{+\infty} Z(t) \mathrm{d} t$, we finally get

$$
D_{s}=D_{s}^{0}-\frac{D_{s}^{0^{2}}}{3 k_{\mathrm{B}} T} \int_{0}^{\infty} \mathrm{d} t\left\langle\mathbf{F}_{s}^{M M}(t) \cdot \mathbf{F}_{s}^{M M}(0)\right\rangle
$$

which is equivalent to (3.14). Thus, the constant term corresponds to the fast dynamics of the solute particles coupled to the motion of the solvent molecules. The Smoluchowski model, which is a continuous solvent model, is not able to give the short time value $D_{s}^{0}$, but the second term, which corresponds to the effect of the ion-ion interactions (relaxation effect).

The resulting time-dependent diffusion coefficient for a simple ion in water is represented in Fig. 8. Mode-coupling theory and Brownian dynamics yield the same results. Self-diffusion coefficients of ions decrease as functions of the concentrations, because the electrostatic friction (relaxation effect) is more important at high concentration. However, this effect takes time. It can be shown that the corresponding relaxation time is the Debye time $\tau_{\mathrm{D}}$. In the example, if $t<100 \mathrm{ps}$, the diffusion coefficient is more than the long-time value, because the electrostatic friction does not have the time to be completely established.

This result can indeed explain the discrepancy between the self-diffusion coefficients measured by time of flight neutron scattering and NMR or tracer methods [81]. The typical time scale of 


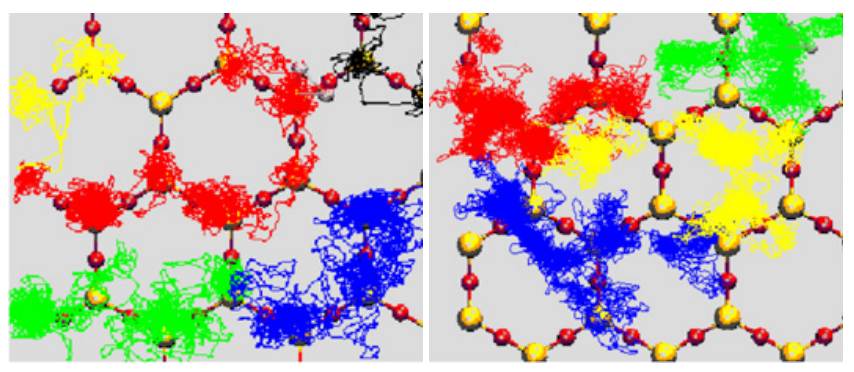

Figure 9. Simulated trajectories of individual $\mathrm{Cs}^{+}$cations (left) and oxygen atoms of individual water molecules (right) confined in a clay interlayer, hydrated to the monolayer state. Trajectories are projected onto the plane of the clay layers, which appear as underlying hexagonal patterns of silicon (yellow) and oxygen (red) atoms.

such neutron scattering experiments is relatively short, typically $20 \mathrm{ps,} \mathrm{which} \mathrm{is} \mathrm{much} \mathrm{less} \mathrm{than} \mathrm{the}$ relaxation time of the ionic atmosphere $\tau_{\mathrm{D}}$. Consequently the values obtained do not take into account the whole relaxation effect. They are found to be greater that those measured by long time methods such as NMR.

The introduction of hydrodynamic interactions in this Brownian approach [82] does not modify the results to a great extent. Hydrodynamic interactions slightly slow down the electrostatic friction, so that the self-diffusion coefficient is slightly greater. Nevertheless, the relaxation time is the same, and it confirms that because of the short times involved in neutron scattering experiments, the self-diffusion measured by that technique can be different from the long-time values. Neutron-scattering is able to look at dynamical processes at an intermediate time scale, similar to the one of the electrostatic relaxation effects involved in electrolyte solutions.

\section{ATOMISTIC DESCRIPTION OF COMPLEX DYNAMICS}

Systems containing severely confined electrolytes deserve the attention of a fully atomistic description, of both the confining matrix and the electrolyte itself. We shall use here the example of low-hydrated clays, where water molecules and cations find themselves in a quasi two-dimensional confinement between adjacent crystalline, negatively charged clay layers (the height of the so-called interlayer is of the order of a few $\AA$ ). Information obtained from classical molecular dynamics simulations on such a system can highlight for example the presence of preferential sites for ions or water on the clay surface [32]. Figure 9 features the trajectories of $\mathrm{Cs}^{+}$cations and oxygen atoms of individual water molecules, projected onto the plane of the clay layers. From the motion of $\mathrm{Cs}^{+}$cations, we observe immediately the preferential sites above clay silicon atoms, which allow a coordination to three oxygen atoms of the underlying clay surface. No such preferential sites are observed for the water molecules themselves, neither are they seen in case of $\mathrm{Na}^{+}$ions in the interlayer (not shown). $\mathrm{Na}^{+}$ions are more tightly hydrated and remain closer to the middle of clay interlayer.

Even for such a severe confinement, in the limit of long times (order of $100 \mathrm{~s}$ of ps to a few ns), the motion of the ions and water molecules never-the-less shows a diffusive behaviour in two dimensions, i.e. in the plane of the clay layers. The resulting two-dimensional diffusion coefficients (obtained via the mean square displacement method) are summarised in Figure 10. In general, at room temperature, for clays hydrated to the so-called monolayer state (a single layer of water molecules in each interlayer), the diffusion coefficients of water are about an order of magnitude smaller than for bulk water, and are not very sensitive to the nature of cation present. Also, regarding the diffusion of the ions, we observe diffusion coefficients of rather similar value for both $\mathrm{Cs}^{+}$and $\mathrm{Na}^{+}$, while the local mechanism seems very different, as highlighted above. The local details of motion therefore do not necessarily have a strong impact on the long term transport coefficients. 


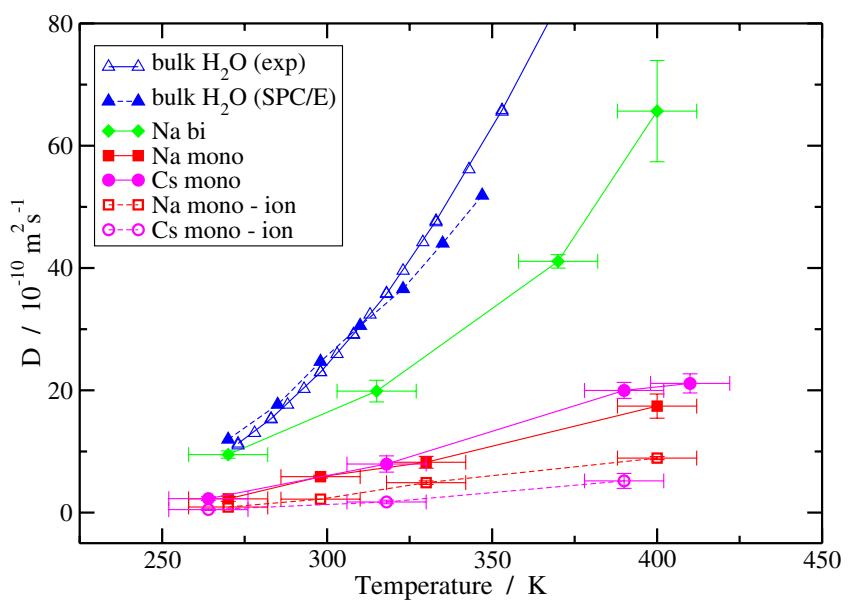

Figure 10. Two-dimensional diffusion coefficients of water and ions confined in clays (diffusion in the plane of the clay layers) as a function of temperature. Three simulated clay systems are presented: $\mathrm{Na}$ and Cs clays hydrated to the monolayer state (Na-mono and Cs-mono) and Na clay hydrated to the bilayer state (Na bi). Three dimensional diffusion coefficients of bulk water, both simulated (SPC/E model of water) and experimental (PF-NMR, [83]) are also shown.

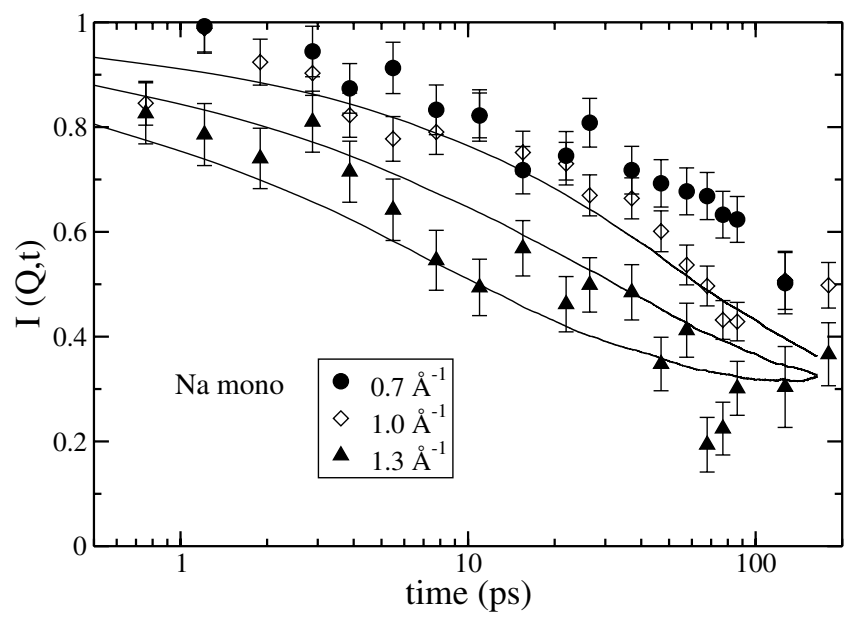

Figure 11. The incoherent intermediate scattering function, $I(Q, t)$, for hydrogen atoms of water molecules confined in a Na clay in the monolayer state, at three different wave-vectors, $Q$, as shown. Symbols refer to neutron scattering measurements (neutron spin echo technique), lines are the results of molecular dynamics simulations.

Neutron scattering and classical molecular dynamics simulations are a powerful combination of experimental and simulation techniques to study ion and water dynamics in complex systems such as clays, e.g. [84-86], or other confining matrices. The strength of neutron scattering comes into play especially when the diffusing species of interest is hydrogen-rich, in comparison to other components of the system. The neutron scattering signal in such cases is dominated by the incoherent scattering from the hydrogen-rich diffuser and the measured time-dependence of this signal is directly related to the self-motion of the diffuser. The time-dependence of the incoherent signal can be also directly computed from the simulated trajectory of the diffuser for direct comparison with neutron scattering. Figure 11 shows such an example for the incoherent intermediate scattering function, $I(Q, t)$, from 
the hydrogen atoms of water molecules in a Na montmorillonite clay hydrated to the monolayer state. The agreement between the simulated and experimental scattering signal can be used to validate the microscopic interaction potentials, upon which the simulation is built. If agreement is found, simulation provides then a wealth of direct information on the local mechanism of the atomic motion, which gives rise to the observed scattering function.

\section{CONCLUSION}

We briefly described the different approaches used to model the dynamics of bulk and confined electrolyte solutions. Every level of description is valid if the variables it models (relevant variables) are slow variables. In the case of hydrodynamics, the macroscopic laws are valid as long as the typical size of the system is larger than a few nanometers, if proper boundary conditions are taken into account. Continuous solvent models are able to describe slow dynamics of ions which can be measured by neutron scattering. They are based on a Brownian model. Nevertheless, for the dynamics of the solvent and the fast processes, molecular dynamics is unavoidable.

\section{References}

[1] J. M. G. Barthel H. Krienke W. Kunz. Physical Chemistry of Electrolyte Solutions. Springer, 1998.

[2] J. B. Hubbard P. G. Wolynes. In R. R. Dogonadze et al., editor, Chemical Physics of Solvation part. D. Elsevier, 1988.

[3] B. Bagchi. Microscopic derivation of the hubbard-onsager-zwanzig expression of imiting ionic conductivity. J. Chem. Phys., 109:3989-3993, 1998.

[4] P. Debye E. Hückel. Phys. Z., 24:305, 1923.

[5] L. Onsager R. M. Fuoss. Irreversible processes in electrolytes. J. Phys. Chem., 36:2689-2778, 1932.

[6] J.-F. Dufrêche O. Bernard S. Durand-Vidal P. Turq. Analytical theories of transport in concentrated electrolyte solutions from the msa. J. Phys.Chem. B, 109:9873, 2005.

[7] W. G. McMillan J. E. Mayer. The statistical mechanics of multicomponents systems solutions. J. Chem. Phys., 13:276, 1945.

[8] H. L. Friedman. A Course in Statistical Mechanics. Prentice-Hall, Englewood Cliffs, New Jersey 07632, 1985.

[9] D. A. McQuarrie. Statistical Mechanics. HarperCollins, 1976.

[10] J.-P. Hansen I. R. McDonald. Theory of Simple Liquids. Academic Press, 1986.

[11] R. A. Robinson R. H. Stokes. Electrolyte Solutions. Butterworths, 1970.

[12] H. L. Friedman. Thermodynamic excess functions for electrolyte solutions. J. Chem. Phys., 32:1351-1562, 1960.

[13] H. L. Friedman. Lewis-randall to memillan-mayer conversion for the thermodynamic excess functions of solutions. part i. partial free energy coefficients. J. Sol. Chem., 1:387-412, 1972.

[14] E. Bich W. Ebeling H. Krienke. Interionishe wechselwirkugen und thermodynamik starker elektrolyte. Z. phys. Chemie, Leipzig, 257:549-562, 1976.

[15] H. Krienke. Zur Statistischen Theorie der Ionenlösungen in Thermodynamischen Gleichgewicht. PhD thesis, Rostock Universität, 1971.

[16] P. Faigl. Ein Beitrag zur Statistischen Thermodynamik verdünnter wässriger Elektrolytlösungen. PhD thesis, Rostock Universität, 1978.

[17] L. Blum J. S. Hø ye. Mean spherical model for asymmetric electrolytes. 2. thermodynamic properties and the pair correlation function. J. Phys. Chem., 81:1311-1316, 1977.

[18] A. P. Lyubartsev A. Laaksonen. Calculation of effective interaction potentials from radial distributions functions: A reverse monte carlo approach. Phys. Rev. E, 52:3730-3737, 1995. 
[19] A. P. Lyubartsev A. Laaksonen. Osmotic and activity coefficients from effective potentials for hydrated ions. Phys. Rev. E, 55:5689-5696, 1997.

[20] A. P. Lyubartsev S. Marcelja. Evaluation of effective ion-ion potentials in aqueous electrolytes. Phys. Rev. E, 65:041202, 2002.

[21] D. C. Brydges Ph. A. Martin. Coulomb systems at low density: a review. J. Stat. Phys., 96:11631330, 1999.

[22] J. J. Molina J.-F. Dufrêche M. Salanne O. bernard M. Jardat P. Turq. Models of electrolyte solutions from molecular descriptions: The example of nacl solutions. Phys. Rev. E, 80:065103(R), 2009.

[23] T. J. Murphy J. L. Aguirre. Brownian motion of $n$ interactiong particules. 1. extension of the einstein diffusion relation to the $n$-particule case. J. Chem. Phys., 57:2098, 1972.

[24] P. M. V. Résibois. Electrolyte Theory. Harper \& Row, 1968.

[25] M. Jardat O. Bernard P. Turq G. R. Kneller. Transport coefficients of electrolyte solutions from smart brownian dynamics simulations. J. Chem. Phys., 110:7993-7999, 1999.

[26] W. Ebeling J. Rose. Conductance theory of concentrated electrolytes in an msa-type approximation. J. Sol. Chem., 10:599-609, 1981.

[27] J.-F. Dufrêche O. Bernard P. Turq. Transport equations for concentrated electrolyte solutions: reference frame, mutual diffusion. J. Chem. Phys., 116:2085, 2002.

[28] E. S. Boek M. Sprik. Ab initio molecular dynamics study of the hydration of a sodium smectite clay. J. Phys. Chem. B, 107:3251, 2003.

[29] D. Tunega M. H. Gerzabek H.Lischka. Ab initio molecular dynamics study of a monomolecular water layer on octahedral and tetrahedral kaolinite surfaces. J. Phys. Chem. B, 108:5930, 2004.

[30] L. Benco D. Tunega J. Hafner et al. Ab initio density functional theory applied to the structure and proton dynamics of clays. Chem. Phys. Lett., 333:479, 2001.

[31] N. T. Skipper G. Sposito F.-R. C. Chang. Clays Clay Miner., 43:294, 1995.

[32] N. Malikova V. Marry J.-F. Dufrêche P. Turq. Temperature effect in a montmorillonite clay at low hydration-microscopic simulation. Mol. Phys., 102:1965, 2004.

[33] M. Arab D. Bougeard K. S. Smirnov. Structure and dynamics of interlayer species in a hydrated zn-vermiculite. a molecular dynamics study. Phys. Chem. Chem. Phys., 6:2446, 2004.

[34] J. A. Greathouse K. Refson G. M. Sposito. Molecular dynamics simulation of water mobility in magnesium-smectite hydrates. J. Am. Chem. Soc., 122:11459, 2000.

[35] P. Turq F. Lantelme H. L. Friedman. Brownian dynamics : its application to ionic solutions. J. Chem. Phys., 66:3039-3044, 1977.

[36] V. Marry J.-F. Dufrêche M. Jardat P. Turq. Equilibrium and electrokinetic phenomena in charged porous media from microscopic and mesoscopic models: electro-osmosis in montmorillonite. Mol. Phys., 101:3111, 2003.

[37] E. Trizac L. Bocquet R. Agra R et al. Effective interactions and phase behaviour for a model clay suspension in an electrolyte. J. Phys. Cond. Matt., 122:11459, 2000.

[38] M. Rosanne M. Paszkuta J.F. Thovert P. Adler. Electro-osmotic coupling in compact clays. Geophys. Research Lett., 31:L18614, 2004.

[39] P. Leroy A. Revil. A triple layer model of the surface electrochemical properties of clay minerals. J. Coll. Int. Science, 270:371, 2004.

[40] C. Moyne M. A. Murad. Electro-chemo-mechanical couplings in swelling clays derived from a micro/macro-homogenization procedure. Int. J. Solids Struct, 39:6159, 2002.

[41] C. Moyne M. A. Murad. Macroscopic behavior of swelling porous media derived from micromechanical analysis. Transport in Porous Media, 50:127, 2003.

[42] S. R. de Groot P. Mazur. Non-Equilibrium Thermodynamics. Dover, 1983.

[43] P. Porion M. Al Mukhtar A. M. Faugere R. J. Pellenq S. Meyer A. Delville. Water self-diffusion within nematic dispersions of nanocomposites: a multiscale analysis of ${ }^{1} \mathrm{~h}$ pulsed gradient spinecho nmr measurements. J. Phys. Chem. B, 107:4012-4023, 2003. 
[44] N. G. Van Kampen. Elimination of fast variables. Phys. Rep., 124:69, 1985.

[45] N. K. Ailawadi A. Rahman R. Zwanzig. Generalized hydrodynamics and analysis of current correlation functions. Phys. Rev. A, 4:1616-1625, 1971.

[46] R. Zwanzig. Nonequilibrium Statistical Mechanics. Oxford University Press, 2001.

[47] V. Marry P. Turq. Microscopic simulations of interlayer structure and dynamics in bihydrated heteroionic montmorillonites. J. Phys. Chem. B, 107:1832, 2003.

[48] J.-F. Dufrêche V. Marry N. Malikova P. Turq. Molecular hydrodynamics for electro-osmosis in clays: from kubo to smoluchowski. J. Mol. Liq, 118:145, 2005.

[49] L. Joly C. Ybert E. Trizac L. Bocquet. Liquid friction on charged surfaces: From hydrodynamic slippage to electrokinetics. J. Chem. Phys., 125:204716, 2006.

[50] H. Risken. The Fokker Planck Equation: Methods of Solutions and Applications. Springer Series in Synergetics, 1996.

[51] N. G. Van Kampen. Stochastic Processes in Physics and Chemistry. North-Holland, 1992.

[52] B. Bagchi. Microscopic expression for dielectric friction on a moving ion. J. Chem. Phys., 95:467-478, 1991.

[53] J. Hubbard L. Onsager. Dielectric dispersion and dielectric friction in electrolyte solutions. i. J. Chem. Phys., 67:4850, 1977.

[54] D. Moroni B. Rotenberg J.-P. Hansen S. Succi S. Melchionna. Solving the fokker-planck kinetic equation on a lattice. Phys. Rev. E, 73:066707, 2006.

[55] B. Rotenberg J.-F. Dufrche B. Bagchi E. Giffaut J.-P. Hansen P. Turq. Ion dynamics in compacted clays: Derivation of a two-state diffusion-reaction scheme from the lattice fokker-planck equation. J. Chem. Phys., 14:154701, 2006.

[56] A. R. Altenberger H. L. Friedman. Theory of conductance and related isothermal transport coefficients in electrolytes. J. Chem. Phys., 78:4162-4173, 1983.

[57] W. Sung H. L. Friedman. Time-correlation functions of the smoluchowski level of description of solutions and suspensions. J. Chem. Phys., 80:2735-2741, 1983.

[58] P. Turq J. Barthel M. Chemla. Transport, Relaxation and Kinetic Processes in Electrolyte Solutions. Springer-Verlag, 1992.

[59] D. L. Ermak. A computer simulation of charged particles in solution. i. technique and equilibrium properties. J. Chem. Phys., 62:4189, 1975.

[60] J. Rotne S. Prager. Variational treatment of hydrodynamic interaction in polymers. J. Chem. Phys., 50:4831-4837, 1969.

[61] H. Yamakawa. Transport properties of chains in dilute solution: Hydrodynamic interaction. J. Chem. Phys., 51:436-443, 1970.

[62] J. Garcia de la Torre V. A. Bloomfield. Hydrodynamic properties of complex, rigid, biological macromolecules : theory and applications. Quat. Rev. Biophys., 14:81, 1981.

[63] B. U. Felderhof R. B. Jones. Linear response theory of sedimentation and diffusion in a suspension of spherical particles. Physica, 119A1:591-608, 1983.

[64] W. Ebeling R. Feistel G. Kelbg R. Sändig. Generalization of onsager semiphenomenological theory of electrolytic conductance. J. Non-Equilib. Thermodyn., 3:11-28, 1978.

[65] P. J. Rossky J. D. Doll H. L. Friedman. Brownian dynamics as smart monte carlo simulation. J. Chem. Phys., 69:4628, 1978.

[66] G. Mériguet E. Dubois M. Jardat A. Bourdon G. Demouchy V. Dupuis B. Farago R. Perzynski P. Turq. Understanding the structure and the dynamics of magnetic fluids: coupling of experiment and simulation. J. Phys. Condens. Matter, 18:S2685-S2696, 2006.

[67] G. Mériguet M. Jardat P. Turq. J. Chem. Phys., 121:6078, 2004.

[68] J. M. Verwey J. Th. G. Overbeek. Theory of the Stability of Lyotropic Colloids. Elsevier, Amsterdam, 1948

[69] E. Waisman J. L. Lebowitz. Mean spherical model integral equation for charged hard spheres. i. method of solution, 2. results. J. Chem. Phys., 56:3086, 1971. 
[70] C. W. Outhwaite V. C. L. Hutson. The mean spherical model for charged hard spheres. Mol. Phys., 29:1521-1531, 1975.

[71] L. Blum. Mean spherical model for asymmetric electrolytes i. method of solution. Mol. Phys., 30:1529-1535, 1975.

[72] L. Blum. Primitive electrolytes in the mean spherical approximation. In H. Eyring D. Henderson, editor, Theoretical Chemistry: Advances and Perspectives, volume 5, pages 1-66. Academic Press, 1980.

[73] W. Ebeling K. Scherwinsky. On the estimation of theoretical individual activity coefficients of electrolytes. Z. phys. Chemie, Leipzig, 264:1-14, 1983.

[74] T. Cartailler P. Turq L. Blum N. Condamine. Thermodynamics of ion association in the mean spherical approximation. J. Phys. Chem., 96:6766-6772, 1992.

[75] O. Bernard W. Kunz P. Turq L. Blum. Conductance in electrolyte solutions using the mean spherical approximation. J. Phys. Chem., 96:3833-3840, 1992.

[76] J.-F. Dufrêche M. Jardat T. Olynyk O. Bernard P. Turq. Mutual diffusion coefficient of charged particles in the solvent-fixed frame of reference from brownian dynamics simulation. J. Chem. Phys., 117:3804-3810, 2002.

[77] O. Bernard W. Kunz P. Turq L. Blum. Self-diffusion in electrolyte solutions using the mean spherical approximation. J. Phys. Chem., 96:398-403, 1992.

[78] A. Chandra B. Bagchi. Ion conductance in electrolyte solutions. J. Chem. Phys., 110:1002410034, 1999.

[79] J.-F. Dufrêche O. Bernard P. Turq A. Mukherjee B. Bagchi. Phys. Rev. Lett., 88:095902, 2002.

[80] R. Kubo. The fluctuation-dissipation theorem. Rev. Prog. Phys., 29:255-284, 1966.

[81] W. Kunz P. Turq M.-C. Bellisent-Funel P. Calmettes. Dynamic and spatial correlations of tetrapentylammonium ions in acetonitrile. J. Chem. Phys., 95:6902-6910, 1991.

[82] J.-F. Dufrêche M. Jardat P. Turq B. Bagchi. Electrostatic relaxation and hydrodynamic interactions for self-diffusion of ions in electrolyte solutions. J. Phys. Chem. B, 112:10264, 2008.

[83] M. Holz S. Heil A. Sacco. Temperature-dependent self-diăusion coefficients of water and six selected molecular liquids for calibration in accurate $1 \mathrm{~h} \mathrm{nmr}$ pfg measurements. Phys. Chem. Chem. Phys., 2:4740-4742, 2000.

[84] N. Malikova A. Cadène V. Marry E. Dubois P. Turq. Diffusion of water in clays on the microscopic scale: Modelling and experiment. J. Phys. Chem. B, 110:3206-3214, 2006.

[85] N. Malikova E. Dubois V. Marry B Rotenberg P. Turq. Dynamics in clys - combining neutron scattering and microscopic simulation. Z. Phys. Chem., 224:153-181, 2010.

[86] V. Marry E. Dubois N. Malikova S. Durand-Vidal S. Longeville J. Breu. Water dynamics in hectorite clays: influence of temperature studied by coupling neutron spin echo and molecular dynamics. Environmenal Science and Technology, accepted. 